\title{
Overtone Mobility Spectrometry: Part 3. On the Origin of Peaks
}

\author{
Stephen J. Valentine, ${ }^{1}$ Ruwan T. Kurulugama, ${ }^{2}$ David E. Clemmer ${ }^{1}$ \\ ${ }^{1}$ Department of Chemistry, Indiana University, Bloomington, IN, 47405, USA \\ ${ }^{2}$ Pacific Northwest National Laboratory, Richland, WA, USA
}

\begin{abstract}
The origin of non-integer overtone peaks in overtone mobility spectrometry (OMS) spectra is investigated by ion trajectory simulations. Simulations indicate that these OMS features arise from higher-order overtone series. An empirically-derived formula is presented as a means of describing the positions of peaks. The new equation makes it possible to determine collision cross sections from any OMS peak. Additionally, it is extended as a means of predicting the resolving power for any peak in an OMS distribution.
\end{abstract}

Key words: Ion mobility spectrometry, Overtone mobility spectrometry, Mass spectrometry

\section{Introduction}

$\mathrm{T}$ he recent application of ion mobility spectrometry (IMS) for the characterization of complex mixtures [114] and the development of hybrid IMS techniques [15-25] has generated interest in improving the resolution of mobility separations. The resolving power $\left(R_{I M S}\right)$ of mobility separations depends on the drift field $(E)$, the drift length $(L)$, and the temperature $(T)$ of the buffer gas according to [26]

$$
\frac{t_{D}}{\Delta t_{D}}=\left(\frac{L E z e}{16 k_{B} T \ln 2}\right)^{1 / 2}
$$

In Equation $1, t_{D}, \Delta t_{D}, k_{b}$, and ze correspond to the drift time of the ion, the full width at half-maximum (FWHM) of the drift time peak, Boltzmann's constant, and the charge of the ion, respectively. Instrumental developments have led to a number of high-resolution drift tube designs exhibiting resolving powers that range from $\sim 100$ to 250 [24, 27-34]. The square root dependence on the parameters: $E, L$, and $T$

Electronic supplementary material The online version of this article (doi:10.1007/s13361-011-0087-y) contains supplementary material, which is available to authorized users.

Correspondence to: David E. Clemmer; e-mail: clemmer@indiana.edu make it difficult to develop higher resolving power instruments with incremental changes in instrument design. For example, doubling the length of the drift region only increases $R_{I M S}$ by a factor of 1.41 .

Recently, the technique of overtone mobility spectrometry (OMS) has been introduced as a means of separating ions $[35,36]$. In this approach, drift fields are applied in a timedependent manner, resulting in the transmission of ions with mobilities that are resonant to the field application frequency. Theoretical considerations led to an equation relating the OMS resolving power $\left(R_{O M S}\right)$ to experimental parameters associated with instrumentation geometry and the IMS resolving power $\left(R_{I M S}\right)$ as shown in Equation 2 [36].

$$
R_{O M S}=\frac{1}{1-\left[1-\frac{C_{2}}{R_{I M S}}\right]\left[\frac{m n-\left[\phi-1-\frac{l_{e}}{l_{t}+l_{e}}\right]}{m n}\right]}
$$

The variables $\Phi, m$, and $n$ correspond to the OMS operational parameters the phase number of the system (number of distinct field applications settings), the field application harmonic number, and the number of OMS drift tube segments, respectively. The variables $l_{e}$ and $l_{t}$ are the lengths of ion elimination and ion transmission regions (see below), respectively. $\mathrm{C}_{2}$ is a constant allowing the comparison to $R_{I M S}$. $R_{O M S}$ is expressed for experimental data as $f / \Delta f$

Received: 15 October 2010

Revised: 15 January 2011

Accepted: 18 January 2011

Published online: 4 March 2011 
where $f$ is the frequency of the peak in the OMS distribution and $\Delta f$ is obtained at FWHM.

Equation 2 shows that $R_{O M S}$ is proportional to $m$ and $n$ indicating that higher resolution separations can be obtained (compared with $R_{I M S}$ ) for incremental changes in instrumentation parameters. Recently, high-resolution OMS separations $\left(R_{O M S}>200\right)$ of gas-phase ubiquitin ions have been reported [37]. Separate experiments employing a circular drift tube operated in an OMS mode yielded peaks with $R_{O M S}>400$ [38]. Such high resolving powers are obtainable because the ions travel around the circular drift tube more than 60 times thereby increasing $n$. Improvements in $R_{O M S}$ come at a cost to overall sensitivity. Ion loss results from the increased selectivity of the mobility filtering process, decreased ion transmission through gridded lenses, and increased ion diffusion for measurements with extended experimental timescales. Thus, high-resolution OMS separations are currently less sensitive than lower-resolution commercial instrumentation employing mobility separations. That said, these examples demonstrate how Equation 2 provides insight that is useful for experimental analyses. Recently, a frequency scanning method for OMS experiments employing a circular drift tube has been demonstrated for high-efficiency separation of components in a tryptic digest mixture [38].

Although Equation 2 estimates $R_{O M S}$ of many peaks, it fails to estimate $R_{O M S}$ values for a series of peaks centered at frequencies that are non-integer multiples of the fundamental transmission frequency $\left(f_{f}\right)$. The largest of these features occurs between the $f_{f}$ peak and the next major harmonic peak. These peaks are often sharp, having $R_{O M S}$ values that are higher than the neighboring major harmonic peaks. In the work presented here, we use ion trajectory simulations to provide insight into the origin of these peaks as well as the factors that contribute to increased $R_{O M S}$. We use this insight to develop a mathematical framework for describing the positions and shapes of all peaks ( $f_{f}$ and overtones). The resulting information suggests that it should be possible to tailor OMS separations to the frequency region in which these higher-resolution overtone peaks are found in order to achieve higher separation efficiency.

\section{Experimental}

\section{OMS Measurements}

General aspects of IMS theory [26, 39-42], instrumentation [17, 18, 22, 24, 43, 44], and techniques [45-47] have been discussed in detail elsewhere. Additionally, OMS separations have been described in detail, including the method of mobility selection, operational modes of OMS devices, and theoretical considerations of $R_{O M S}$ [36]. Briefly, an OMS instrument contains a segmented drift tube. Adjoining drift segment $(d)$ regions are interfaced by two lenses with wire mesh grids. Drift fields are applied across a number of adjacent segments; this number represents the phase $(\Phi)$ of the system and also corresponds to the number of unique field settings employed in the separation. By periodically applying the drift field to adjacent segments and shifting the field application by one $d$ region after each period, ion elimination regions $\left(d_{e}\right)$ are generated between a number of lenses with grids; these ion elimination regions shift with each field application setting. Ions that have mobilities allowing them to traverse one $d$ region [consisting of one ion transmission region $\left(d_{t}\right)$ as well as one $d_{e}$ region] during one field application time period, are selected for transmission through the OMS device. Ions with mismatched mobilities eventually locate in one of the $d_{e}$ regions and are neutralized on the grids. In this manner the OMS device imposes a mobility filter on ions from a continuous source. An OMS spectrum is obtained by scanning the field application frequency while recording the ion current.

For the experiments reported here, OMS spectra have been recorded using different OMS drift tube designs. OMS datasets for $[\mathrm{M}+\mathrm{Na}]^{+}$raffinose ions have been recorded for instruments employing different field application phases ( $\Phi=2$ to 6 ). Although the total drift tube length has been maintained at $\sim 1.8 \mathrm{~m}$, the lengths of individual $d$ regions $\left(l_{t}+l_{e}\right)$ have been altered to allow the use of the same $n$ (22) for each $\Phi$ setting. A field application frequency range of $\sim 500$ to $\sim 50,000 \mathrm{~Hz}$ has been used for all experiments.

\section{Raffinose Samples}

Raffinose (90\% purity) was obtained from Sigma Aldrich and used without further purification. A raffinose sample $\left[0.2 \mathrm{mg} \cdot \mathrm{mL}^{-1}\right.$ in water:acetonitrile solution $(50 \%: 50 \%$ by volume) with $2 \mathrm{mM} \mathrm{NaCl}$ ] was infused through a pulledtip capillary at a flow rate of $0.30 \mu \mathrm{L} \cdot \mathrm{min}^{-1}$. The capillary was maintained at a DC potential that was $2.2 \mathrm{kV}$ above the ESI source region entrance. $[\mathrm{M}+\mathrm{Na}]^{+}$raffinose ions are desolvated in the ESI source region and are transmitted via an electrodynamic ion funnel $[48,49]$ into the drift tube where the OMS separation is performed as described above.

\section{OMS Ion Trajectory Simulations}

Ion trajectory simulations for OMS systems with $\Phi=2$ to 6 have been performed. The algorithm used for these calculations was developed in house and is described elsewhere [24, 50]. Only a brief introduction to the algorithm is given here. The calculations utilize two-dimensional field arrays similar to those generated in SIMION [51] to determine time-dependent ion displacements. The displacement of an ion is the sum of the ion motion contributions from the mobility of the ion $(K)$ as well as its diffusion. The former contribution is field dependent [i.e., the drift velocity $\left(v_{D}\right)$ is proportional to the product of the mobility and the electric field $(K E)$ ] [39], whereas the latter 
is a randomized contribution in the algorithm (discussed below).

The current algorithm is versatile in that it allows different OMS phase systems to be examined. Figure 1 shows a schematic of the OMS device used for the ion trajectory simulations and a cartoon diagram of the initial ion placement in the OMS device with the field array settings for different phase systems. A $\Phi=2$ system requires two field arrays, including one in which the oddnumbered $\left(1,3,5\right.$, etc.) $d_{e}$ regions contain fields that will transmit ions; the remaining, even-numbered $d_{e}$ regions contain fields that will cause the elimination of ions. For the second field array, the transmission and elimination $\left(d_{e}\right)$ regions are reversed. Initially, the algorithm selects one field array and reads in a region (4 field array points) that surrounds the two-dimensional position of the ion for which the calculation is being performed. A weightedaverage field is then calculated for both dimensions based on the distance of the ion to the field values provided at each surrounding array point. The displacement due to the ion mobility is then calculated for a user-defined time step (typically $\leq 2 \mu \mathrm{s}$ ) by multiplying by $v_{D}$. For these simulations, $K$ is taken from an experimentally available value (e.g., the mobility of $[\mathrm{M}+\mathrm{H}]^{+}$bradykinin ions).
To simulate random diffusion in two dimensions, the root mean square displacement of an ion, $\sqrt{\overline{r^{2}}}$, is converted into a polar coordinate vector by randomizing a single angle $(\Theta)$. The $y$ - and $z$-axis vector components are determined

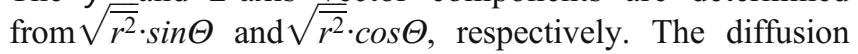
value is then added to the displacement due to the ions mobility to provide a net ion displacement for each time increment. A total of $n=11$ segments have been used for each of the ion trajectory simulations. For the simulations of the $\Phi=2$ system, the first two $d_{t}$ regions and the first $d_{e}$ region (164 total grid units along the z-axis of the field array) are filled with ions (10 ions at each grid unit). The conjoining $d_{e}$ regions are each 5 grid units wide (i.e., $l_{e}=5$ grid units). A complete analysis for a specific frequency requires 1640 separate ion trajectory simulations.

The simulations for the $\Phi=3$ system require three discrete field array files and the first three $d$ region segments are filled with ions up to the $d_{e}(3)$ region (Figure 1). Therefore, the total number of grid units filled with ions is 249. The total number of grid units that are filled with ions for the $\Phi=4, \Phi=5$, and $\Phi=6$ systems are 334, 419, and 504 , respectively. Also, the simulations for the $\Phi=4, \Phi=5$, and $\Phi=6$ systems require 4,5 , and 6 independent field array files, respectively. For all simulations, the starting (a)
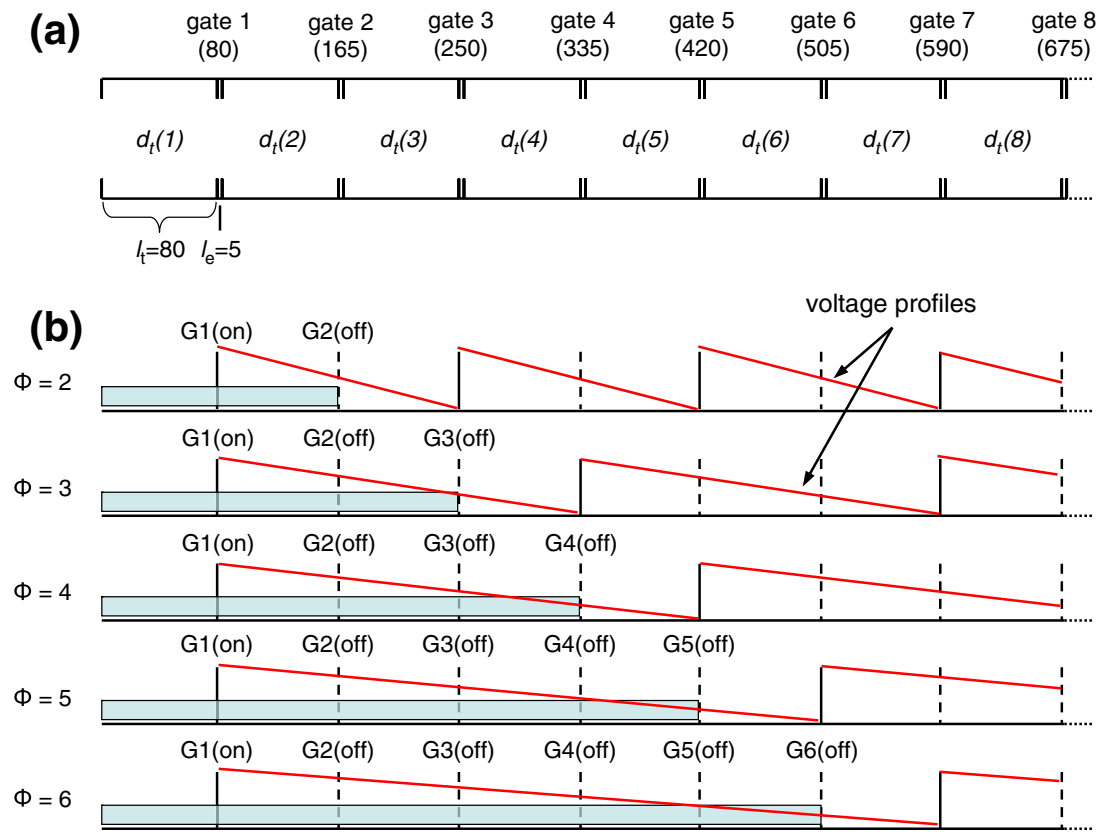

Figure 1. (a) Schematic diagram of the OMS device for the ion trajectory simulation. The first eight ion transmission $\left(d_{t}\right)$ and ion elimination regions $\left(d_{e}\right)$ are shown. For these simulations, the OMS device utilized consists of 11 segments. Each segment is divided into 80 grid units and the conjoining $d_{e}$ sections consist of 5 grid units. Ten ions are placed at each grid unit for the simulation. The number in parenthesis under the gate number corresponds to the starting grid unit for each gate region. (b) $A$ cartoon diagram showing the initial ion placement for ion trajectory simulations using different phase systems. Each simulation is started by turning on the first gate (G1 or first $d_{e}$ region), therefore, all the ions placed in the first segment get neutralized at the fundamental frequency level. Red lines indicate the "sawtooth" voltage profile for the field application depicted with the gate settings [35-37]. For example, gates $(n \Phi+1$ where $n=0,1,2,3, \ldots)$ that show an abrupt change (minimum to maximum value in the sawtooth profile) are designated as $d_{e}$ regions for this field setting indicating the location of ion neutralization. The solid blue lines show the numbers of $d$ regions that are filled by the ion beam prior to initiating ion elimination at G1. This filling size is related to values for $R_{O M S}$ as well as intensities of specific OMS dataset features (see text and Figure 4 for details) 
frequency was $500 \mathrm{~Hz}$ and the frequency is stepped by $100 \mathrm{~Hz}$ for each analysis. Using a maximum frequency of $50 \mathrm{kHz}$, a total of 495 separate analyses for each value of $\Phi$ have been performed. Ion intensities at each frequency setting are determined as the percentage of ions in the simulation that are transmitted through the entire OMS region. OMS spectra generated from the ion trajectory simulations are compared with experimental datasets for which the same phase has been employed.

\section{Results and Discussion}

\section{Dependence of OMS Distribution Peaks on $\Phi$}

Figure $2 \mathrm{a}$ shows OMS spectra recorded for $[\mathrm{M}+\mathrm{Na}]^{+}$ raffinose ions for different $\Phi$ values. For $\Phi=2$, a feature is observed at $\sim 2050 \mathrm{~Hz}$ corresponding to transmission of the $[\mathrm{M}+\mathrm{Na}]^{+}$raffinose ions at the $f_{f}$. Two other major features are also observed at field application frequencies that are 3 and 5 times the $f_{f}$. Major OMS distribution peaks such as these have been related to $\Phi$ using $m$ according to the relationship

$$
m=\Phi(h-1)+1
$$

$[35,36]$

where $h$ corresponds to a harmonic index $(h=1,2,3 \ldots)$. Features in OMS distributions are delineated by their frequencies $(f)$ as they relate to the $f_{f}$ according to

$$
f=m f_{f}
$$

From the expression for $m$ (Equation 3), major harmonic peaks in OMS distributions obtained from a $\Phi=3$ system would be centered at $\sim 4 f_{f}, \sim 7 f_{f}, \sim 10 f_{f}$, etc. Figure 2 a shows the presence of this harmonic series in the OMS spectra for $[\mathrm{M}+\mathrm{Na}]^{+}$raffinose ions collected with a $\Phi=3$ device. Figure $2 \mathrm{a}$ also shows that lower-frequency peaks from the major harmonics series predicted by $m$ are also observed for the $\Phi=4$, the $\Phi=5$, and the $\Phi=6$ systems. Peak assignments for the major harmonics series as observed in the experimental datasets (Figure $2 \mathrm{a}$ ) are listed in Table 1.

Examination of the OMS spectra for the $[\mathrm{M}+\mathrm{Na}]^{+}$ raffinose ions in Figure 2a shows that many other features that cannot be accounted for by values of $m$ (as defined by Equation 3) are present. For the $\Phi=4$ system, two distinct features are observed between the $f_{f}$ and the $5 f_{f}$ peaks. These features have peak centers of 4691 and $6049 \mathrm{~Hz}$ which are factors of 2.28 and 2.95 times higher than the $f_{f}$. It is instructive to consider the resolution of these peaks. $R_{O M S}$ values of 47 and 41 have been determined for these two features. These values are greater than those determined for the neighboring major harmonic peaks (i.e., the peaks centered at $f_{f}$ and $5 f_{f}$ ). Peaks located between the first two major harmonic peaks are observed for all systems with the exception of the $\Phi=$
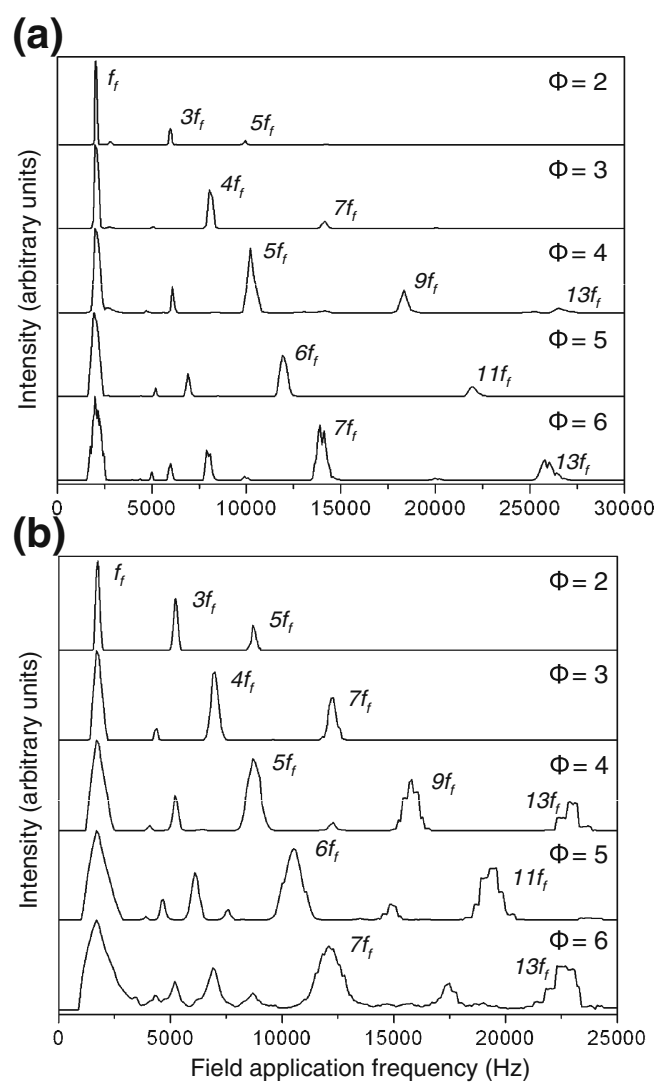

Figure 2. (a) OMS distributions obtained for $[\mathrm{M}+\mathrm{Na}]+$ raffinose ions using OMS devices employing $\Phi=2$ to 6 systems. Peaks in the primary harmonic series (see text for discussion) are labeled for each system. For these studies, the OMS drift tube is $\sim 1.8 \mathrm{~m}$ long and a buffer gas pressure and drift field of $\sim 3$ Torr and $10 \mathrm{~V} \cdot \mathrm{cm}^{-1}$ have been used. The OMS region of the drift tube is $\sim 1.2$ Torr and a total number of $22 d$ regions have been employed for each system. b) OMS spectra obtained using ion trajectory simulations for OMS devices employing $\Phi=2$ to 6 systems. Ion trajectory simulations are carried out using an algorithm developed in house (see text for details). Peaks in the primary harmonic series are labeled for each system. For this virtual OMS device, $11 d$ regions have been employed. A model mobility of $0.09 \mathrm{~m}^{2} \cdot \mathrm{V}^{-1} \cdot \mathrm{s}^{-1}$ has been used for all ions in the simulations described here

2 system, and the number of peaks in this region increases with increasing values of $\Phi$ (Figure 2a). These experimental data also show that some additional peaks arise between the second and third major harmonic peaks of the OMS distributions. Examples include peaks centered at $\sim 14198$ and $\sim 20216 \mathrm{~Hz}$ for the $\Phi=4$ and the $\Phi=6$ systems, respectively. These peak centers are factors of 6.92 and 9.86 higher than the $f_{f}$, which are also not predicted by integer values of $m$ according to Equation 4. These overtone peaks all have $R_{O M S}$ values exceeding those of neighboring major harmonic peaks. These overtone peaks (not described previously) with associated peak intensities are also listed in Table 1 . 
Table 1. OMS distribution peak assignments

\begin{tabular}{|c|c|c|c|c|c|c|c|c|c|}
\hline \multicolumn{5}{|c|}{ A. Experimental distributions ${ }^{\mathrm{a}}$} & \multicolumn{5}{|c|}{ B. Ion trajectory simulations ${ }^{\mathrm{b}}$} \\
\hline$\Phi^{\mathrm{c}}$ & Frequency $^{\mathrm{d}}$ & Intensity ${ }^{\mathrm{e}}$ & $m^{\mathrm{f}}$ & $h^{\mathrm{g}}$ & $\Phi^{\mathrm{c}}$ & Frequency $^{\mathrm{d}}$ & Intensity ${ }^{\mathrm{e}}$ & $m^{\mathrm{f}}$ & $h^{\mathrm{g}}$ \\
\hline 2 & 2050 & $1.00^{\circ}$ & 1 & 1 & 2 & 1742 & 1.00 & 1 & 1 \\
\hline 2 & 6019 & 0.20 & 3 & 2 & 2 & 5233 & 0.58 & 3 & 2 \\
\hline 2 & 9938 & 0.07 & 5 & 3 & 2 & 8738 & 0.28 & 5 & 3 \\
\hline 3 & 2050 & 1.00 & 1 & 1 & 3 & 1747 & 1.00 & 1 & 1 \\
\hline 3 & 5031 & 0.03 & 2.5 & 1.5 & 3 & 4358 & 0.13 & 2.5 & 1.5 \\
\hline 3 & 8056 & 0.48 & 4 & 2 & 3 & 6988 & 0.76 & 4 & 2 \\
\hline 3 & 14198 & 0.12 & 7 & 3 & 3 & 9597 & 0.00 & 5.5 & 2.5 \\
\hline 4 & 2050 & 1.00 & 1 & 1 & 3 & 12239 & 0.47 & 7 & 3 \\
\hline 4 & 4691 & 0.03 & 2.33 & 1.33 & 4 & 1755 & 1.00 & 1 & 1 \\
\hline 4 & 6049 & 0.34 & 3 & 1.5 & 4 & 4073 & 0.05 & 2.33 & 1.33 \\
\hline 4 & 10340 & 0.75 & 5 & 2 & 4 & 5240 & 0.39 & 3 & 1.5 \\
\hline 4 & 14198 & 0.03 & 7 & 2.5 & 4 & 6411 & 0.01 & 3.67 & 1.67 \\
\hline 4 & 18457 & 0.27 & 9 & 3 & 4 & 8721 & 0.80 & 5 & 2 \\
\hline 4 & 26790 & 0.08 & 13 & 4 & 4 & 12243 & 0.09 & 7 & 2.5 \\
\hline 5 & 2050 & 1.00 & 1 & 1 & 4 & 15763 & 0.57 & 9 & 3 \\
\hline 5 & 5185 & 0.10 & 2.66 & 1.33 & 4 & 22986 & 0.31 & 13 & 4 \\
\hline 5 & 6975 & 0.27 & 3.5 & 1.5 & 5 & 1760 & 1.00 & 1 & 1 \\
\hline 5 & 12068 & 0.48 & 6 & 2 & 5 & 3822 & 0.04 & 2.25 & 1.25 \\
\hline 5 & 22037 & 0.12 & 11 & 3 & 5 & 4656 & 0.23 & 2.67 & 1.33 \\
\hline 6 & 2050 & 1.00 & 1 & 1 & 5 & 6109 & 0.53 & 3.5 & 1.5 \\
\hline 6 & 4383 & 0.02 & 2.2 & 1.2 & 5 & 7565 & 0.12 & 4.33 & 1.67 \\
\hline 6 & 5000 & 0.12 & 2.5 & 1.25 & 5 & 8200 & 0.01 & 4.75 & 1.75 \\
\hline 6 & 6019 & 0.20 & 3 & 1.33 & 5 & 10463 & 0.79 & 6 & 2 \\
\hline 6 & 8025 & 0.34 & 4 & 1.5 & 5 & 13472 & 0.02 & 7.67 & 2.33 \\
\hline 6 & 10031 & 0.07 & 5 & 1.67 & 5 & 14940 & 0.17 & 8.5 & 2.5 \\
\hline 6 & 14136 & 0.68 & 7 & 2 & 5 & 16300 & 0.01 & 9.33 & 2.67 \\
\hline 6 & 20216 & 0.03 & 10 & 2.5 & 5 & 19369 & 0.58 & 11 & 3 \\
\hline 6 & 26173 & 0.25 & 13 & 3 & 5 & 23790 & 0.02 & 13.5 & 3.5 \\
\hline & & & & & 6 & 1757 & 1.00 & 1 & 1 \\
\hline & & & & & 6 & 4316 & 0.18 & 2.5 & 1.25 \\
\hline & & & & & 6 & 5196 & 0.36 & 3 & 1.33 \\
\hline & & & & & 6 & 6910 & 0.50 & 4 & 1.5 \\
\hline & & & & & 6 & 8656 & 0.21 & 5 & 1.67 \\
\hline & & & & & 6 & 12138 & 0.71 & 7 & 2 \\
\hline & & & & & 6 & 14622 & 0.07 & 8.5 & 2.25 \\
\hline & & & & & 6 & 15636 & 0.07 & 9 & 2.33 \\
\hline & & & & & 6 & 17483 & 0.29 & 10 & 2.5 \\
\hline & & & & & 6 & 18859 & 0.07 & 11 & 2.67 \\
\hline & & & & & 6 & 22747 & 0.50 & 13 & 3 \\
\hline
\end{tabular}

${ }^{\mathrm{a}}$ Data obtained from experimental results shown in Figure 2a

${ }^{\mathrm{b}}$ Peaks obtained from the ion trajectory simulations shown in Figure $2 \mathrm{~b}$

${ }^{\mathrm{c}}$ OMS system phase indicating the number of unique field application settings

${ }^{\mathrm{d}}$ Peak maxima in $\mathrm{Hz}$ obtained from the experimental and theoretical plots (Figure 2)

${ }^{\text {e}}$ Peak intensities are reported as a fraction of the base peak intensity

${ }^{\mathrm{f}}$ Values of $m$ are estimated based on multiples of the $f_{f}(m=1)$. These values are confirmed with Equation 6

${ }^{\mathrm{g}}$ Values of $h$ are obtained from Equation 3 using values of $m$ and $\Phi$ given in the table

This assessment above does not include the very small peak observed at $\sim 2727 \mathrm{~Hz}$ in the $\Phi=2$ system that also appears to be present in other systems (Figure 2a). We note that this feature may arise as another overtone peak for $[\mathrm{M}+$ $\mathrm{Na}]^{+}$raffinose ions or it may result from an isobaric ion. In favor of the latter argument is the fact that this feature is observed at the same position in systems of higher value, behaving in a manner similar to the $f_{f}$ peak for the $[\mathrm{M}+\mathrm{Na}]^{+}$ raffinose ions. The theoretical treatment described here accounts for all OMS features for this sample with the exception of this small peak. Additionally, many of the peaks in the $\Phi=6$ system are observed to resemble multiplet features. It is suggested that this peak splitting results from an artifact of the measurement (e.g., ion source and wavedriver fluctuations or imperfections in the $\Phi=6$ system design). Such an explanation is favored as evidence for peak splitting is not observed in the other systems (Figure 2a) and is also not observed-apart from the undersampling of frequencies - in the simulations (Figure 2b). We also note that all other OMS experiments at appropriate overtone frequencies provide distributions that are similar to those obtained with IMS measurements [35-37]; for [M $+\mathrm{Na}]^{+}$ raffinose ions, this results in a single dataset feature.

OMS distributions obtained from ion trajectory simulations are shown in Figure $2 \mathrm{~b}$. Table 1 also contains a complete list of peaks observed in these distributions. Overall, the OMS distributions derived from the simulations are very similar to experimental distributions obtained using the same OMS systems. For example, for the $\Phi=2$ system, only the $f_{f}$, the $3 f_{f}$, and the $5 f_{f}$ harmonic peaks are observed. For the $\Phi=3,4,5$, and 6 systems, major harmonics are also observed. However, for these higher systems additional 
peaks are observed between the major harmonic peaks exhibiting overtones matching those observed experimentally. The relative peak intensities of the different peaks are also similar to the experimental results. For all systems, peak intensities decrease with increasing frequency within the major harmonic peak series. Conversely, in general, peak intensities increase with increasing frequency for the overtone peaks observed between the major harmonic peaks.

It is instructive to consider a single system in greater detail in order to obtain a general description of peaks observed in OMS experiments. The $\Phi=4$ system generated four primary harmonic peaks (Figure $2 \mathrm{~b}$ ) centered at 1755 , $8721,15,763$, and $22,986 \mathrm{~Hz}$ and four more low intensity peaks centered at 4073, 5240, 6411, and 12,243 Hz. The four primary harmonic peaks correspond to those observed at the $f_{f}, 5 f_{f}, 9 f_{f}$, and $13 f_{f}$ field application frequencies, respectively, and are predicted for the $\Phi=4$ system by Equation 3. The respective intensities for the major harmonic peaks are $70,56,40$, and 22 . The minor overtone peaks are located at $\sim 2.3 f_{f}, \sim 3 f_{f}, \sim 3.6 f_{f}$, and $\sim 7 f_{f}$, respectively. The intensities for the minor overtone peaks (in order of increasing frequency) are 3.8, 27, 0.7, and 6.2.

Although, the OMS spectra obtained from the ion trajectory simulations resemble those obtained experimentally for $[\mathrm{M}+$ $\mathrm{Na}]^{+}$raffinose, several differences are observed. OMS peaks are more highly resolved in the experimental datasets. This is due to the use of an instrument with 22 segments whereas the ion trajectory simulations employed a model device with 11 segments $[35,36]$. Although the primary harmonic peaks observed for the experimental data are essentially the same as the simulation data, the peak centers are shifted slightly due to differences in the mobilities of raffinose and the theoretical ion used in the simulations. Although the OMS spectra obtained for the $\Phi=3, \Phi=4$, and $\Phi=5$ systems show minor peaks centered at similar overtone values compared with the experimental data, a greater number of minor overtone peaks is observed for the simulation data. For example, for a $\Phi=5$ system, four and two peaks are observed between the first two major harmonic peaks for the simulation and the experimental data, respectively. Finally, it is noted that a greater degree of ion transmission is observed for the ion trajectory simulations. Most likely this is a result of the fewer gates used in the ion trajectory simulations resulting in fewer ions lost due to diffusion. Additionally, the simulations are carried out in an ideal environment and do not account for perturbations in experimental conditions such as the presence of fringing fields that may affect the transmission of ions in the OMS drift tube.

The peak profile for the $\Phi=6$ system obtained for [M+ $\mathrm{Na}]^{+}$raffinose ions is significantly different from the data obtained using the ion trajectory simulations. This is primarily due to the low-resolution OMS spectrum obtained for the computer simulation. The baseline for the simulation is not zero at any point (Figure 2b), indicating that ions leak through the virtual OMS device. This is likely due to the use of an insufficient $n$; that is, a larger number of $d$ region segments would result in increased OMS separation effi- ciency as reported earlier and predicted by Equation 2 [36]. This also suggests that there is a minimum number of $d$ regions required to prevent ion leakage. Below we relate the selectivity of OMS devices to their ability to segment the continuous ion beam into discrete, transmittable packets of ions. Large transmission packets generated by the $\Phi=6$ system result in less discrimination of ions based on mobilities (see discussion below). Selectivity can be increased by introducing more $d$ regions as observed previously in OMS experiments [35-37]; the greater number of $\mathrm{d}$ regions more effectively divides the continuous ion beam (see discussion below and supplementary information). Thus, ion trajectory simulations employing a greater $n$ would produce distributions that are more similar to the experimental datasets.

\section{Generation of $f_{f}$ Peaks in OMS Distributions}

During the course of an OMS separation, the continuous ion beam is divided into whole regions that are either transmitted through the drift tube or eliminated due to the application of alternating field settings in the segmented drift tube [36]. Figure 3 shows the distance traversed (final grid number) by ions at different starting positions (initial grid number) in the ion trajectory simulations for the $\Phi=2,3,5$, and 6 systems. Simulations are carried out using a field application frequency representing maximum ion transmission (i.e., the $f_{f}$, which ranges from $\sim 1700$ to $\sim 1750$ for these systems). Figure 3 shows which ions (with respect to initial position) are neutralized and which pass through the OMS device. The grid number values provided on the y axis can be related to the gate numbers given in Figure 1 to provide locations for ion neutralization events. The last grid number, 1015 , corresponds to gate 12 . Ions that reach gate 12 , have passed through the OMS device unaffected. For example, the plot for $\Phi=2$ shows that all the ions up to a starting grid number of 85 have been neutralized at some point within the OMS device while almost all the ions starting after grid number 85 propagate through the OMS region.

It is instructive to consider the starting positions of ions that are neutralized on grids after the initial gates. For all systems these ions are those that are located closer to the edges of the transmitted portion of the ion beam. For example, the transmission plot for the $\Phi=2$ system in Figure 3 shows that the ion transmission region extends from $\sim 85$ to $\sim 165$ grid units. However, ion losses are observed to occur on the edges of this transmission region from $\sim 85$ to $\sim 100$ and from $\sim 152$ to $\sim 160$ grid units. Ion loss occurs because of the diffusion of ions into the $d_{e}$ regions during the simulation. The $\Phi=2$ system shows a duty cycle of $\sim 50 \%$; that is, neglecting the ions lost due to diffusion, roughly half of the initial ion population reaches the end gate. Simulations show that this $50 \%$ duty cycle is true even for overtone peaks in the $\Phi=2$ system. Plots for the $\Phi=3$, $\Phi=5$, and $\Phi=6$ systems (Figure 2) show duty cycles of $66 \%, 80 \%$, and $83 \%$, respectively. Examination of these data 

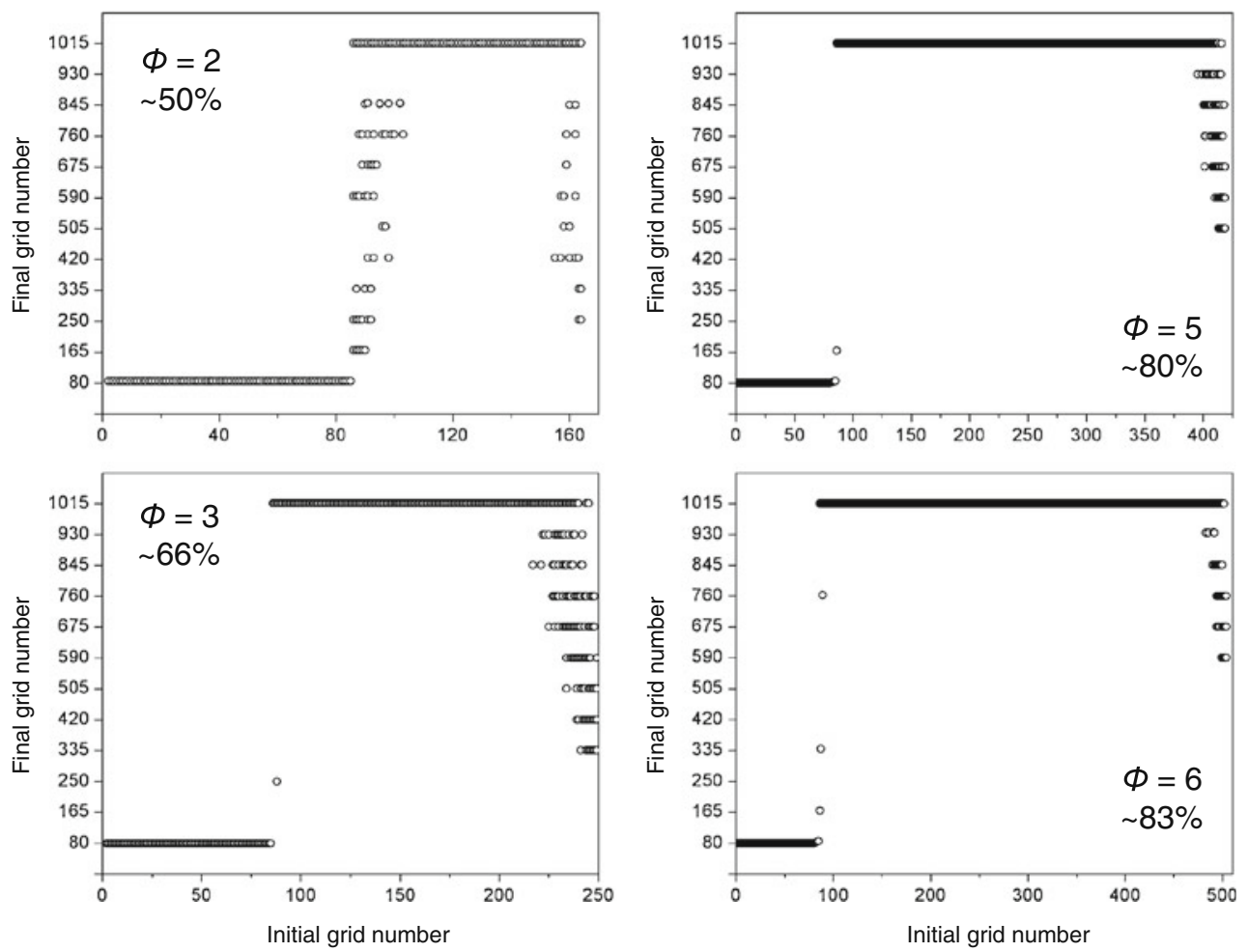

Figure 3. Plots of the final grid number versus the initial grid number for $\Phi=2,3,5$, and 6 systems. The ion trajectory simulations have been carried out at the $f_{f}$ for the model molecule (see Figure 2). lons that reach a grid unit of 1015 are considered to have been transmitted through the entire OMS device. lons shown at other positions indicate neutralization events at the respective gate (Figure 1). The estimated duty cycle for each phase system is provided

as well as that of the $\Phi=4$ system suggests that the duty cycle (expressed as a percentage) for a given system can be estimated by $[(\Phi-1) / \Phi] \times 100[35-37]$.

To understand the origin of the $f_{f}$ peaks and the dependence of $R_{O M S}$ values on $\Phi$ for these peaks, it is necessary to consider the transmission of ions in an OMS device in more detail. Figure 4 a shows the transmitted portions of the continuous ion beam for a $\Phi=4$ system using a field application frequency that is equal to the $f_{f}$. During the first field application time period, the first gate (G1 in Figure 4) is set to eliminate ions from the continuous ion beam. Upon activating the second field setting, ions from the continuous source fill the second $d$ region. Because of the resonance in mobility and field application frequency, when the leading edge of the ion beam reaches $\mathrm{G} 2$, the field is reset to transmit ions into the third $d$ region. Similarly, the ions are transmitted through G3 into the fourth $d$ region. At this point, ion elimination reverts to G1 and creates a "gap" in the ion beam. This effectively generates a transmitted portion of the ion beam covering $\sim 3 d$ regions. The size of this transmitted portion of the ion beam directly influences the associated $R_{O M S}$.

In describing the effect of transmitted portion size on $R_{O M S}$, consider the analysis of a mixture of ions. Here each transmitted portion of the ion beam (Figure 4a) would have ions with differing mobilities. Those with resonant mobilities would traverse exactly one $d$ region in one field application setting and thus be transmitted through the entire drift tube as part of the transmitted portion of the ion beam. Ions with mobilities not matching the field application frequency would gradually move toward the edge of the transmitted portion of the ion beam during the course of the measurement. If the mismatch is sufficiently large, such ions would eventually be eliminated in a preceding or trailing $d_{e}$ region. Because an ion with a mismatched mobility may travel close to the edge of the transmitted portion of the beam during the course of a measurement and still be transmitted to the detector, the size of the transmitted portion of the beam limits the size of achievable $R_{O M S}$. A larger size of the transmitted portion of the ion beam allows a greater mismatch in mobilities of transmitted ions and results in lowered $R_{O M S}$. This is evidenced in small $R_{O M S}$ values for $f_{f}$ peaks in OMS distributions as Figure 4a shows a relatively large transmitted portion of the ion beam for these operating conditions. Previously we have shown that the transmitted portions of the ion beam increase with increasing values of $\Phi$. This accounts for the higher resolution of systems with smaller $\Phi$ (Figure 2) [36].

\section{Generation of OMS Peaks in Overtone Regions}

To describe the origin of overtone peaks it is useful to first consider the major harmonics predicted by Equation 3 . Figure $4 \mathrm{~b}$ shows the transmitted portion of the continuous ion beam for a $\Phi=4$ system using a field application 
frequency that is equal to $5 f_{f}$. Here, G1 is set to initially block the transmission of ions into the second $d$ region. When the field settings are switched, G1 allows transmission into the second $d$ region. However, the transmitted portion of the beam (assuming all ions with mobilities in resonance with the $f_{f}$ ) only travels $1 / 5$ of the way into the second $d$ region before the field switches again (using the $5 f_{f}$ settings). With each field application, the ion beam travels another $1 / 5$ of the $d$ region. On the fourth field application setting, the $d_{e}$ region has returned to $\mathrm{G} 1$ and the ion beam is cut off creating a smaller "gap" ( $1 / 5$ of the $d$ region) in the ion beam. Figure $4 \mathrm{~b}$ shows that the transmitted portion of the ion beam is $\sim 3 / 5$ of the $d$ region in length. This is much smaller (5-fold) than the $\sim 3 d$ region length of the transmitted portion of the ion beam generated under the $f_{f}$ operational conditions (Figure 4a). Previous work has shown that $R_{O M S}$ values scale directly with field application frequency $[35,36]$. Figure $4 \mathrm{a}$ and $\mathrm{b}$ show the origin of this dependence. Because the transmitted portion of the ion beam using the $5 f_{f}$ settings is $\sim 5$-fold smaller than that of the $f_{f}$ settings, the degree of allowed mobility mismatch in a mixture of ions should be about 5 times less resulting in the observed difference in $R_{O M S}$ values.

The examples above provide a physical basis for the origin of the major harmonic peaks (including the $f_{f}$ ) in OMS distributions. That is, portions of the ion beam are transmitted in specific frequency regions while they are not transmitted in other frequency regions. A similar mechanism of ion beam trimming can be used to explain the origin of the features observed between the major harmonic peaks. Consider the peak at $5240 \mathrm{~Hz}$ for the $\Phi=4$ system in Figure $2 \mathrm{~b}$. This peak is lower in intensity than the surrounding major harmonic peaks $\left(f_{f}\right.$ and $\left.5 f_{f}\right)$ and has been assigned as the $3 f_{f}$ overtone. Figure $4 \mathrm{c}$ shows the process of generating transmitted portions of the continuous ion beam while operating the OMS device at $3 f_{f}$. Here, the transmitted portion of the ion beam traverses $1 / 3$ of the distance of a $d$ region with each successive field application setting. Overall, the process generates transmitted portions of the ion beam that are $1 / 3$ of a $d$ region in length. This smaller length (compared with the $f_{f}$ and $5 f_{f}$ operational modes) results in increased $R_{O M S}$ value. The smaller length also accounts for the relative peak height of this feature as a smaller transmitted portion of the ion beam results in a greater susceptibility to ion loss due to diffusion.

Figure 4 effectively shows that the origin of all peaks in OMS distributions arise from differences in the manner in which the continuous ion beam is divided into transmitted portions. This process accounts not only for the observed peaks for different OMS devices but also for the observed $R_{O M S}$ values and peak intensities. Although Figure 4 presents a simple scheme for the overall process, we note that it represents ideal conditions. The ion trajectory simulations carried out for a $\Phi=4$ system can be used to support the proposed physical process responsible for the generation of peaks in OMS distributions. Plots of the ion transmission efficiency for each feature for this system $(\Phi=$ 4 in Figure 2b) are shown as Supplementary Figure 1. Here, the process of ion diffusion is accounted for. Overall, the generation of transmitted portions of the continuous ion beam of the same relative sizes is confirmed by the ion trajectory simulations.

From the ion trajectory simulations, observations of $d_{e}$ regions involved in the creation of the transmitted portions of the ion beam provide clues into the different types of overtone series present in the different systems. For example, for the primary harmonic series observed in the $\Phi=4$ system (Figure 2b), only the first four $d_{e}$ regions are involved with the creation of the ion transmission packets. That is, ions located in yet to be created ion beam gap regions are neutralized in one of the first four $d_{e}$ regions. However, for the $3 f_{f}$ and $7 f_{f}$ overtone peaks the first five gates are responsible for creation of the ion transmission packets while for the $2.3 f_{f}$ overtone peak the first six $d_{e}$ regions are required. Therefore, it is suggested that there are three different overtone series in an OMS spectrum obtained from a $\Phi=4$ system. The first of these is derived from the primary harmonic series where peak positions are described by $m$ according to Equation 3. These peaks are centered at $f_{f}, 5 f_{f}, 9 f_{f}, 13 f_{f}$, etc. The next series is termed the secondary overtone series with peaks centered at $3 f_{f}$ , $7 f_{f}$, etc. The last group is the tertiary overtone series that produces peaks centered at $2.3 f_{f}, 3.6 f_{f}$, etc.

\section{Comparison of Peaks Generated by Different OMS Systems ( $\Phi)$}

Having described the origin of different peaks in OMS distributions, it is instructive to consider the relationship of the same overtone peaks generated by different OMS systems. Here, we compare the transmitted portions of the ion beam for the $3 f_{f}$ features observed in the $\Phi=2$ and $\Phi=4$ systems (Figure 2). Ion trajectory simulations indicate that the transmitted portions of the ion beam are the same size for this feature in both systems as shown in Supplementary Figure 2. This explains the similarity in $R_{O M S}$ for these two features. However, the number of ion gates required to generate these portions are 2 and 5 for the $\Phi=2$ and $\Phi=4$ systems, respectively. A second comparison can be made between the $5 f_{f}$ features observed in the $\Phi=2$ and $\Phi=4$ systems (Figure 2b). The simulations show (Supplementary Figure 2) that the transmitted portions of the ion beam are 2 times smaller for the $\Phi=2$ system (Figure $2 b$ ). This is the reason for the increased $R_{O M S}$ value associated with this feature as well as its decreased intensity resulting from increased susceptibility to ion loss associated with diffusion.

\section{A Formula for the Prediction of Peak Centers in OMS Distributions}

Previously it has been shown that the primary harmonic peaks are designated by the field application frequency harmonic $m$ according to Equation 3 . For the primary 

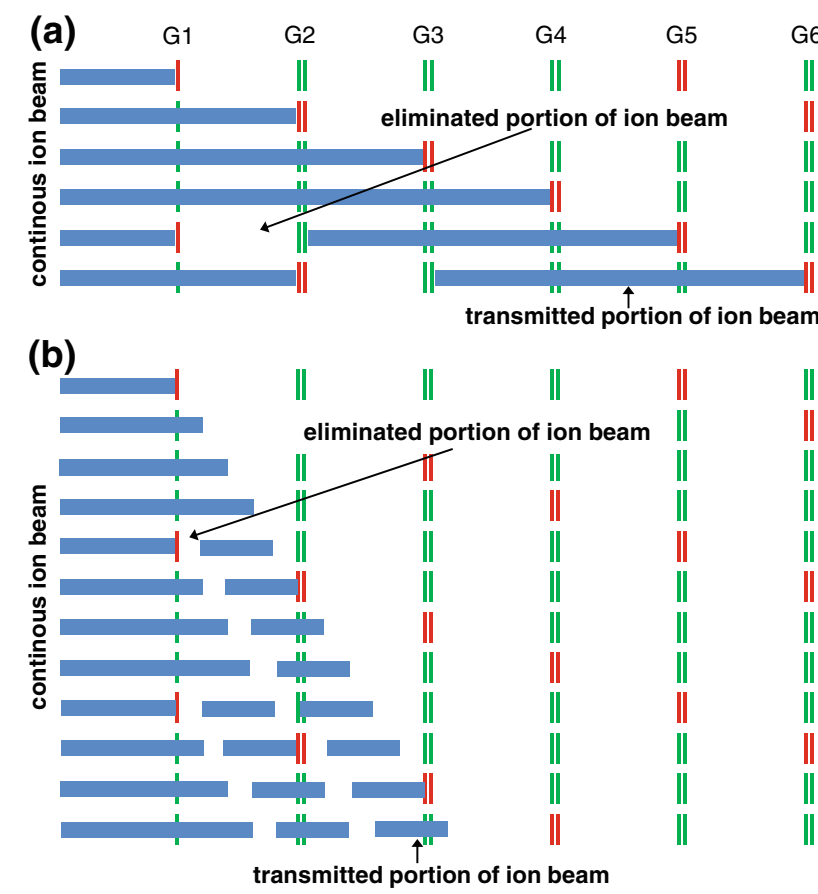

\section{$5 f_{f}$}
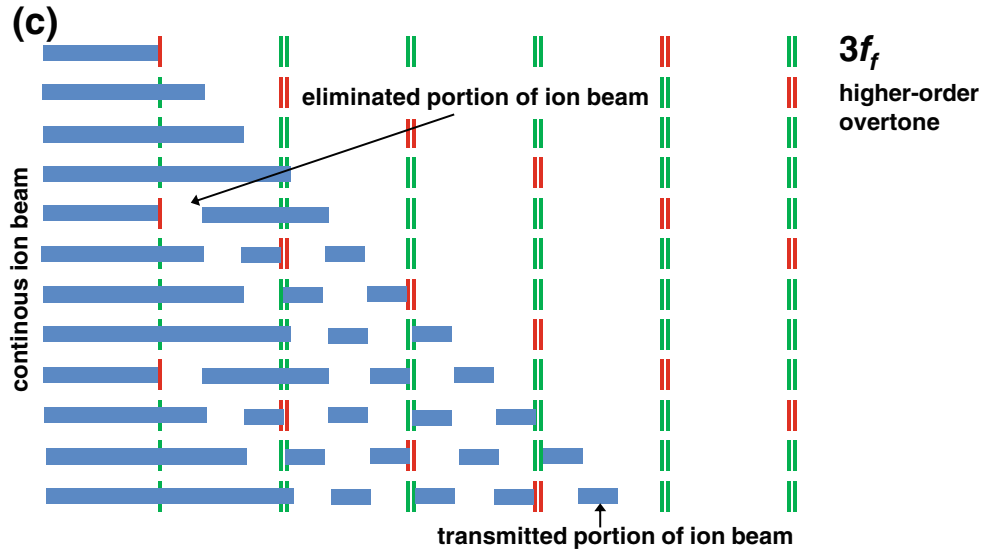

Figure 4. (a) Cartoon representing transmission of ions from an ion beam generated from a single molecular species using a $\Phi=4$ system. The scheme represents transmission at the $f_{f}$ of the ions. Each row represents a separate, sequential voltage setting (red lines in Figure 1). The first line depicts a field application setting in which G1 serves to eliminate ions such that the ion beam does not propagate into the second $d$ region. Application of the next voltage setting (row 2) allows ions to pass into the second $d$ region. The next field setting (row 3 ) allows passage of ions into the third $d$ region and the subsequent field setting (row 4 ) allows the filling of the fourth $d$ region. The next field application (row 5), is a duplicate of the original setting (row 1) and ions are eliminated at G1 creating a "gap" in the original ion beam. This leads to a transmitted portion of the ion beam that is $\sim 3 d$ regions in length. (b) Cartoon representing ion transmission of ions from an ion beam generated from a single molecular species using a $\Phi=4$ system. The scheme represents transmission at the $5 f_{f}$ of the ions. The first row depicts a field application setting in which G1 serves to eliminate ions such that the ion beam does not propagate into the second $d$ region. Application of the next three voltage settings (rows $2-4$ ) allows ions to fill a portion of the second $d$ region (1/5 of the distance with each successive setting). The next field application (row 5 ), is a duplicate of the original setting (row 1) and ions are eliminated at G1 creating a "gap" (1/5 a $d$ region in length) in the original ion beam. This leads to a transmitted portion of the ion beam that is $\sim 3 / 5$ of a $d$ region in length. Subsequent voltage settings (next few rows) show the propagation of the transmitted portion of the ion beam as well as the generation of other transmitted portions of the same size. (c) Cartoon representing ion transmission of ions from an ion beam generated from a single molecular species using a $\Phi=$ 4 system. The scheme represents transmission at the $3 f_{f}$ of the ions. The first row depicts a field application setting in which G1 serves to eliminate ions such that the ion beam does not propagate into the second $d$ region. Application of the next three voltage settings (rows 2-4) allows ions to fill a portion of the second $d$ region (1/3 of the distance with each successive setting). The next field application (row 5), is a duplicate of the original setting (row 1) and ions are eliminated at G1 creating a "gap" (1/3 a $d$ region in length) in the original ion beam. This leads to an initial transmitted portion of the ion beam that is $\sim 1 d$ region in length. However, the next field application setting (row 6) eliminates the middle third of this transmitted portion of the ion beam. The end result is to create portions that are $1 / 3$ of a $d$ region in length. Subsequent voltage settings (next few rows) show the propagation of the transmitted portions of the ion beam 
harmonic series within all systems, $h$ varies as $1,2,3, \ldots$ etc. However, separate calculations are required to describe the other overtone series (hereafter described as higher-order overtones). These can be obtained from the estimated values of $m$ for these higher-order overtones listed in Table 1. For example, for the secondary overtone series, $h$ must vary as $1.5,2,2.5, \ldots$ etc., and for the tertiary overtone series, $h$ must vary as $1.33,1.67,2, \ldots$ etc. The secondary overtone series is observed for systems where $\Phi \geq 3$ and the tertiary overtone series is observed for systems where $\Phi \geq 4$ (Table 1). For a $\Phi=5$ system, in addition to the above overtone series, another series with $h$ varying as $1.25,1.5,1.75, \ldots$ etc, exists. Similarly, for the $\Phi=6$ system, in addition to the overtone series described above, there is another series with $h$ varying as $1.2,1.4,1.6,1.8, \ldots$ etc.

The empirically-derived equation accounting for the higher order overtone series is,

$$
h=\left(\frac{q}{\phi-k}\right)+1
$$

In Equation 5, the variable $k$ (overtone series index) has limits of 0 to $\Phi-1$, and the variable $q$ (overtone peak index) has limits of $0 \rightarrow \infty$. Equations 3 and 5 can be combined to provide a complete index of frequencies $(m)$ for all OMS dataset features according to

$$
m=\phi\left(\frac{q}{\phi-k}\right)+1
$$

Here $m$ no longer corresponds to true harmonics (i.e., integer multiples of the $f_{f}$ ). Thus the new term for $m$ is OMS frequency coefficient.

\section{Determining Ion Collision Cross Sections from OMS Peaks}

Previously, an equation has been reported that allows the determination of collision cross sections using the field application frequencies of the major harmonic peaks [36]. Calculations of collision cross section using OMS data have previously been shown to be very accurate as values obtained from OMS datasets have been determined to be within $\pm 1 \%$ of established values determined by IMS measurements [37]. It is possible to incorporate the variable $m$ into the collision cross section equation to obtain the expression,

$$
\begin{aligned}
\Omega= & \frac{(18 \pi)^{1 / 2}}{16} \frac{z e}{\left(k_{b} T\right)^{1 / 2}}\left[\frac{1}{m_{I}}+\frac{1}{m_{B}}\right]^{1 / 2} \frac{E m}{f\left(l_{t}+l_{e}\right)} \frac{760}{P} \\
& \times \frac{T}{273.2} \frac{1}{N}
\end{aligned}
$$

In Equation 7, the variables $m_{I}$ and $m_{B}$ correspond to the masses of the ion and the buffer gas, respectively. The variables $T, P$, and $N$ correspond to the temperature, the pressure, and the neutral number density at STP of the buffer gas, respectively. Finally, $f$ corresponds to the frequency of the peak used for the calculation.

One advantage of Equation 7 is that it allows the determination of collision cross section from any peak in the OMS dataset; previously this calculation was only valid for primary harmonic peaks. For sample mixtures, OMS spectra can be examined for spectral regions containing limited interference from chemical noise in order to obtain a collision cross section measurement. Additionally, the multiple measurements obtained from the various overtone peaks can serve to confirm collision cross section calculations in order to increase the accuracy of the determination.

A disadvantage of the cross section calculation is that it requires a knowledge of the value of $m$ in order to perform the calculation which can be problematic for sample mixtures. That said, because of the relationship among features in the different peak series, assignments of $m$ may be obtained by direct comparison of related peaks. Such a process would be very similar to that employed in deciphering electrospray ionization (ESI) charge state distributions. Here, a ratio of peak frequencies would be compared to a ratio of $m$ values within an overtone series. One advantage for the OMS approach is that OMS distributions provide even more distinctive information than ESI charge state distributions. This information is contained within the relative intensities of the peaks in that specific overtone series have characteristic intensities. As described in detail above, these intensities are related to the size of the transmitted portion of the ion beam at a given frequency and thus ultimately to $R_{O M S}$. In practice it would be relatively straightforward to determine target OMS regions based on the characteristic mobilities of mixture components. ${ }^{9}$ From there the determination of peak ratios can be performed; as evident in Figure 2, determining features that belong to the same overtone series which are candidates for the ratio comparison is a straightforward process.

\section{Predicting $R_{\text {OMS }}$ for OMS Distribution Peaks}

With an expression describing the frequencies for all expected peaks in OMS distributions, a question arises as to whether or not $R_{O M S}$ can be predicted for peaks of different overtone series. Peak widths in OMS distributions indicate that Equation 6 cannot be substituted directly into Equation 2 as this does not capture the fact that $R_{O M S}$ values for peaks in higher-order overtone series are larger than those of neighboring primary harmonic peaks. For example, from ion trajectory simulations of the $\Phi=4$ system (Figure 2b), the peaks centered at $f_{f}$ and $5 f_{f}$ have $R_{O M S}$ values of 3.4 and 14 , respectively. The first observable peaks in the higher-order overtone series at $\sim 2.33 f_{f}$ and $\sim 3 f_{f}$ have $R_{O M S}$ values of 30 and 20 , respectively. Thus, although $R_{O M S}$ increases with increasing frequency for 
the primary harmonic series, for the higher-order overtone series, it decreases with increasing frequency. This is only true for higher-order overtone peaks that have the same value of $q$ (Equation 6). For example, a $q$-value of 1 is determined for the peaks at $\sim 2.33 f_{f}$ and $\sim 3 f_{f}$ (Figure 2 b). A low-intensity peak centered at $\sim 3.67 f_{f}$ is also located between the $f_{f}$ peak and the $5 f_{f}$ peak. This peak however has a $R_{O M S}$ value of $\sim 50$. The difference is that a different $q$ value ( 2 in this case) describes this peak position according to Equation 6.

An observation that helps to yield an expression for $R_{O M S}$ for higher-order overtone series peaks is that the ratios of $R_{O M S}$ values for the peak of interest and the major harmonic peak with the same $q$ value can be related by the fraction $\Phi /(k+1)$ where $k$ is given as the value used to calculate the position of the peak of interest. For example, for the ion trajectory simulations of the $\Phi=4$ system, the peaks centered at $2.33 f_{f}$ $(q=1, k=1)$ and $5 f_{f}(q=1, k=3)$ have $R_{O M S}$ values of 30 and 14 , respectively. The ratio of $R_{O M S}$ values is 2.14 (30/14). Using a value of $k=1$ for the peak centered at $2.33 f_{f}$, a similar value of 2.0 is obtained for the fraction $\Phi /(k+1)$. This correlation between $R_{O M S}$ ratios and the fraction $\Phi /(k+1)$ is verified for other peaks in higher-order overtone series. For example, the $R_{O M S}$ ratio for the peak centered at $3 f_{f}(q=1, k=2)$ and the peak centered at $5 f_{f}(q=1, k=3)$ is 1.36 . The fraction $\Phi /(k+1)$ for the peak centered at $3 f_{f}$ is 1.33 . This relationship also holds for OMS peaks in higher-order overtone series where $q$ is greater than 1. For example, the $R_{O M S}$ ratio for the peak centered at $3.67 f_{f}(q=2, k=1)$ and the peak centered at $9 f_{f}(q=2, k=3)$ is 2.13. The fraction $\Phi /(k+1)$ for the peak centered at $3.67 f_{f}$ is 2.00. Thus, it is suggested that Equation 2 can be modified to provide the $R_{O M S}$ value for major harmonic peaks at a given $q$ value. The resulting equation can then be multiplied by the fraction $\Phi /(k+1)$ to obtain the resolving power of any peak in an OMS distribution. The new $R_{O M S}$ equation becomes

$$
R_{O M S}=\frac{1}{1-\left[1-\frac{C_{2}}{R_{I M S}}\right]\left[\frac{[\phi q+1] n-\left[\phi-1-\frac{l_{e}}{l_{l}+l_{e}}\right]}{[\phi q+1] n}\right]} \frac{\phi}{k+1}
$$

It is instructive to consider the relative agreement between predicted $R_{O M S}$ values and those obtained from the ion trajectory simulations as well as the experimental data shown in Figure 2. Figure 5a shows the comparison of predicted $R_{O M S}$ values (from Equation 8) and $R_{O M S}$ values obtained from the simulation of a $\Phi=4$ system. In general, the $R_{O M S}$ predicted by Equation 8 is very similar to the value obtained from the simulation data (Figure 2b); the average agreement in $R_{O M S}$ is $5.7 \% \pm 3.2 \%$. $R_{O M S}$ values obtained from Equation 8 have also been compared to experimental values. Figure $5 \mathrm{~b}$ shows the comparison of predicted $R_{O M S}$ values and $R_{O M S}$ values obtained from the analysis of $[\mathrm{M}+\mathrm{Na}]^{+}$raffinose ions using a $\Phi=6$ system (Figure $2 \mathrm{a}$ ). As with the simulation data, relatively good agreement in $R_{O M S}$ value is obtained (e.g., on average values agree to within $6.0 \% \pm 5.2 \%$ ). It is somewhat expected that Equation 8 would perform better for the simulation data as such analyses are carried out under ideal conditions. That is, the greater difference in comparison with the experimental data may result in part from variability associated with the instrumentation (pressure, fields, frequencies, etc.). As an example, the high electric field used to neutralize ions in the $d_{e}$ region may partially penetrate into the $d_{t}$ regions affecting the mobilities of the ions. As a final note regarding Equation 8, the method is only valid using minimum and maximum values of $q$ and $k$, respectively, to delineate the

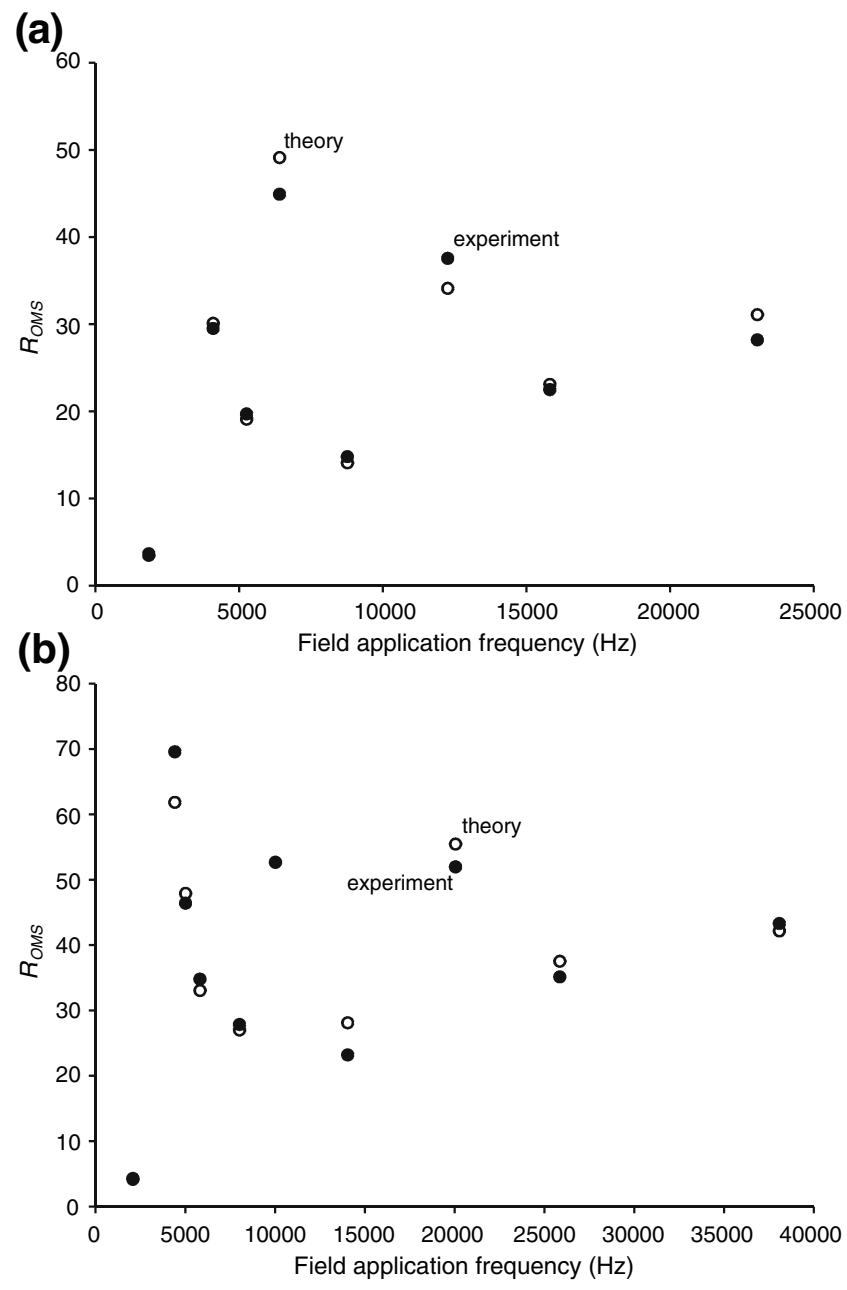

Figure 5. (a) Plot of $R_{O M S}$ values obtained from ion trajectory simulation data (closed circles) and theoretical calculations (open circles) as a function of field application frequency. $R_{O M S}$ values for ion trajectory simulations have been obtained from the data shown in Figure 2b for a $\Phi=4$ system. Theoretical calculations have been performed using Equation 8 for the same peaks from the simulations. (b) Plot of $R_{O M S}$ values obtained from an experimental OMS dataset (closed circles) and theoretical calculations (open circles) as a function of field application frequency. Experimental $R_{O M S}$ values have been obtained for peaks observed in an OMS distribution obtained for $[\mathrm{M}+\mathrm{Na}]^{+}$raffinose ions (Figure $2 \mathrm{a}$ ). The OMS device used to collect the data is a $\Phi=6$ system. Theoretical calculations have been performed using Equation 8 for the same peaks from the simulations 
specific overtone peaks as multiple combinations of these variables can lead to the same $m$ number (Equation 6).

\section{Utilizing the Higher-Order Overtone Region}

Because of the high resolving power afforded by the higherorder overtone regions of OMS distributions, it is instructive to consider practical applications for their use in OMS separations. First, the knowledge gained from ion trajectory simulations can be used to devise high-resolution OMS separations. For example, an OMS instrument with an increased number of $d$ regions can provide substantially higher resolving power. If $n$ is increased from 22 to 100 to create an OMS drift tube that is $\sim 4.5 \mathrm{~m}$ long, the predicted $R_{O M S}$ value for the first overtone peak $(q=1, k=0)$ located between the first two major harmonic peaks would be $>450$ for a $\Phi=6$ system. The development of such instrumentation for routine analyses may have a significant impact for the study of complex mixtures containing ions with similar mobilities including mixtures of molecular isomers.

Related instrumental development work may include the incorporation of higher-order overtone region characterization with a circular drift tube. Recently we have shown that a circular drift tube can be operated in an OMS mode to provide a high resolution separation $\left(R_{O M S}>400\right)$ of mixture components according to differences in mobilities at the resonant $f_{f}$ settings [38]. The current geometry of the circular drift tube is not conducive to transmission of ions at overtone frequencies because the $d_{e}$ region is larger than the $d_{t}$ region. However, it would be relatively straightforward to decrease the size of the $d_{e}$ region such that overtone frequencies could be attained. Assuming transmission of ions for $603 / 4$ cycles as has been performed previously, a theoretical $R_{O M S}>1600$ could be obtained for the first overtone peak $(q=1, k=0)$ using a $\Phi=6$ system.

A separate advantage in using the higher-order overtone region of an OMS distribution is that the separation region is condensed. For example, the higher-order overtone region for a $\Phi=6$ system in which all peaks have a $q$ value of 1 extends from $2 f_{f}$ to $4 f_{f}$. To obtain a similar resolving power in the major harmonics peak region, it would be necessary to scan the frequency range of the $19 f_{f}$ to the $25 f_{f}$ region. Assuming a $f_{f}$ of $2000 \mathrm{~Hz}$, the frequency range to be scanned in the higher-order overtone series is $\sim 2000 \mathrm{~Hz}$; the range increases to $\sim 12,000 \mathrm{~Hz}$ for the major harmonics region. Thus there is an analytical throughput advantage that is quite significant. This advantage can be offset by scanning at a faster rate in the higher frequency region (albeit at a loss to overall ion signal levels) as well as by increasing the step size of the field application frequency settings (leading to less resolution than can be achieved with smaller steps). That said, because of the presence of multiple higher-order overtone peaks in the higher-order overtone series region, a problem may arise from chemical noise. However, because OMS analyses are in effect measurements of collision cross sections, it would be relatively straightforward to determine the overtones for interfering peaks and disregard them in the analysis of the OMS distributions.

\section{Summary and Conclusion}

Ion trajectory simulations have been used to provide an explanation for the origin of peaks in OMS distributions. Simulations show the origin of a major harmonic series of peaks as well as several higher-order overtone series of peaks. From the analysis of different OMS systems, an empirically-derived formula is presented that predicts the peak pattern for any OMS distribution. This formula also allows the determination of ion collision cross sections from any feature within an OMS distribution. Additionally, an analytical expression has been derived that can be used to predict $R_{O M S}$ values as well as to estimate relative peak intensities for expected features in OMS distributions. This information should be valuable in the process of constructing improved OMS instruments. A unique feature of OMS compared with other mobility-based separation techniques is the fact that the analysis can be carried out at different frequency ranges in order to tailor the type of analysis performed to the desired separation efficiency.

\section{Acknowledgments}

The authors are grateful for partial support of this work from the National Institutes of Health (1RC1GM090797-01) and the METACyt initiative, funded by a grant from the Lilly Endowment. This work is also supported in part by funding through the DoD NSWC Crane "Next Generation Threat Detection" (N00164-08-C-JQ11).

\section{References}

1. Moon, M.H., Myung, S., Plasencia, M., Hilderbrand, A.E., Clemmer, D.E.: Nanoflow LC/ion mobility/CID/TOF for proteomics: analysis of a human urinary proteome. J. Proteome Res. 2, 589-597 (2003)

2. Taraszka, J.A., Kurulugama, R., Sowell, R., Valentine, S.J., Koeniger, S.L., Arnold, R.J., Miller, D.F., Kaufman, T.C., Clemmer, D.E.: Mapping the proteome of drosophila melanogaster: analysis of embryos and adult heads by LC-IMS-MS methods. J. Proteome Res. 4, 1223-1237 (2005)

3. Valentine, S.J., Plasencia, M.D., Liu, X., Krishnan, M., Naylor, S., Udseth, H.R., Smith, R.D., Clemmer, D.E.: Toward plasma proteome profiling with ion mobility-mass spectrometry. J. Proteome Res. 5, 2977-2984 (2006)

4. Liu, X., Valentine, S.J., Plasencia, M.D., Trimpin, S., Naylor, S., Clemmer, D.E.: Mapping the human plasma proteome by SCX-LCIMS-MS. J. Am. Soc. Mass Spectrom. 18, 1249-1264 (2007)

5. Fenn, L.S., McLean, J.A.: Simultaneous glycoproteomics on the basis of structure using ion mobility-mass spectrometry. Mol. Biosyst. 5(11), 1298-1302 (2009)

6. Baker, E.S., Livesay, E.A., Orton, D.J., Moore, R.J., Danielson, W.F., Prior, D.C., Ibrahim, Y.M., LaMarche, B.L., Mayampurath, A.M., Schepmoes, A.A., Hopkins, D.F., Tang, K.Q., Smith, R.D., Belov, M. E.: An LC-IMS-MS platform providing increased dynamic range for high-throughput proteomic studies. J. Proteome Res. 9(2), 997-1006 (2010)

7. Dwivedi, P., Wu, P., Klopsch, S.J., Puzon, G.J., Xun, L., Hill, H.H.: Metabolic profiling by ion mobility mass spectrometry (IMMS). Metabolomics 4(1), 63-80 (2008)

8. Kaplan, K., Dwivedi, P., Davidson, S., Yang, Q., Tso, P., Siems, W., Hill, H.H.: Monitoring dynamic changes in lymph metabolome of 
fasting and fed rats by electrospray ionization-ion mobility mass spectrometry (ESI-IMMS). Anal. Chem. 81(19), 7944-7953 (2009)

9. Fenn, L.S., McLean, J.A.: Biomolecular structural separations by ion mobility-mass spectrometry. Anal. Chem. Bioanal. Chem. 39, 905-909 (2008)

10. Trimpin, S., Tan, B., Bohrer, B.C., O’Dell, D.K., Merenbloom, S.I., Pazos, M.X., Clemmer, D.E., Walker, J.M.: Profiling of phospholipids and related lipid structures using multidimensional ion mobility spectrometry-mass spectrometry. Int. J. Mass Spectrom. 287, 58-69 (2009)

11. Isailovic, D., Kurulugama, R.T., Plasencia, M.D., Stokes, S.T., Kyselova, Z., Goldman, R., Mechref, Y., Novotny, M.V., Clemmer, D.E.: Profiling of human serum glycans associated with liver cancer and cirrhosis by IMS-MS. J. Proteome Res. 7(3), 1109-1117 (2008)

12. Plasencia, M.D., Isailovic, D., Merenbloom, S.I., Mechref, Y., Clemmer, D.E.: Resolving and assigning N-linked glycan structural isomers from ovalbumin by IMS-MS. J. Am. Soc. Mass Spectrom. 19 (11), 1706-1715 (2008)

13. Becker, C., Qian, K., Russell, D.H.: Molecular weight distributions of asphaltenes and deasphaltened oils studied by laser desorption ionization and ion mobility mass spectrometry. Anal. Chem. 80(22), 85928597 (2008)

14. Fernandez-Lima, F.A., Becker, C., McKenna, A.M., Rodgers, R.P., Marshall, A.G., Russel, D.H.: Petroleum crude oil characterization by IMS-MS and FTICR MS. Anal. Chem. 81(24), 9941-9947 (2009)

15. Liu, Y., Clemmer, D.E.: Characterizing oligosaccharides using injectedion mobility/mass spectrometry. Anal. Chem. 69, 2504-2509 (1997)

16. Liu, Y., Valentine, S.J., Counterman, A.E., Hoaglund, C.S., Clemmer, D.E.: Injected-ion mobility analysis of biomolecules. Anal. Chem. 69, 728A (1997)

17. Hoaglund-Hyzer, C.S., Li, J., Clemmer, D.E.: Mobility labeling for parallel CID of ion mixtures. Anal. Chem. 72, 2737-2740 (2000)

18. Gillig, K.J., Ruotolo, B., Stone, E.G., Russell, D.H., Fuhrer, K., Gonin, M., Schultz, A.J.: Coupling high-pressure MALDI with ion mobility/ orthogonal time-of flight mass spectrometry. Anal. Chem. 72, 3965$3971(2000)$

19. Valentine, S.J., Kulchania, M., Srebalus Barnes, C.A., Clemmer, D.E.: Multidimensional separations of complex peptide mixtures: a combined high-performance liquid chromatography/ion mobility/time-of-flight mass spectrometry approach. Int. J. Mass Spectrom. 212, 97-109 (2001)

20. Srebalus Barnes, C.A., Hilderbrand, A.E., Valentine, S.J., Clemmer, D. E.: Resolving isomeric peptide mixtures: a combined HPLC/ion mobility-TOFMS analysis of a 4000-component combinatorial library. Anal. Chem. 74, 26-36 (2002)

21. Valentine, S.J., Koeniger, S.L., Clemmer, D.E.: A split-field drift tube for separation and efficient fragmentation of biomolecular ions. Anal. Chem. 75, 6202-6208 (2003)

22. Tang, K., Shvartsburg, A.A., Lee, H.N., Prior, D.C., Buschbach, M.A., Li, F.M., Tolmachev, A.V., Anderson, G.A., Smith, R.D.: High-sensitivity ion mobility spectrometry/mass spectrometry using electrodynamic ion funnel interfaces. Anal. Chem. 77, 3330-3339 (2005)

23. Tang, K.Q., Li, F.M., Shvartsburg, A.A., Strittmatter, E.F., Smith, R.D.: Two-dimensional gas-phase separations coupled to mass spectrometry for analysis of complex mixtures. Anal. Chem. 77(19), 6381-6388 (2005)

24. Koeniger, S.L., Merenbloom, S.I., Valentine, S.J., Jarrold, M.F., Udseth, H.R., Smith, R.D., Clemmer, D.E.: An IMS-IMS analogue of MS-MS. Anal. Chem. 78, 4161-4174 (2006)

25. Merenbloom, S.I., Koeniger, S.L., Bohrer, B.C., Valentine, S.J., Clemmer, D.E.: Improving the efficiency of IMS-IMS by a combing technique. Anal. Chem. 80(6), 1918-1927 (2008)

26. Revercomb, H.W., Mason, E.A.: Theory of plasma chromatography gaseous electrophoresis-review. Anal. Chem. 47, 970-983 (1975)

27. Dugourd, Ph, Hudgins, R.R., Clemmer, D.E., Jarrold, M.F.: Highresolution ion mobility measurements. Rev. Sci. Instrum. 68, 1122-1129 (1997)

28. Wu, C., Siems, W.F., Asbury, G.R., Hill, H.H.: Electrospray ionization high-resolution ion mobility spectrometry-mass spectrometry. Anal. Chem. 70, 4929-4938 (1998)
29. Asbury, G.R., Hill, H.H.: Evaluation of ultrahigh resolution ion mobility spectrometry as an analytical separation device in chromatographic terms. J. Microcolumn Sep. 12, 172-178 (2000)

30. Tang, K., Shvartsburg, A.A., Lee, H., Prior, D.C., Buschbach, M.A., Li, F., Tomachev, A., Anderson, G.A., Smith, R.D.: High-sensitivity ion mobility spectrometry/mass spectrometry using electrodynamic ion funnel interfaces. Anal. Chem. 77, 3330-3339 (2005)

31. Merenbloom, S.I., Koeniger, S.L., Valentine, S.J., Plasencia, M.D., Clemmer, D.E.: IMS-IMS and IMS-IMS-IMS/MS for separating peptide and protein fragment ions. Anal. Chem. 78, 2802-2809 (2006)

32. Gillig, K.J., Ruotolo, B.T., Stone, E.G., Russell, D.H.: An electrostatic focusing ion guide for ion mobility-mass spectrometry. Int. J. Mass Spectrom. 239, 43-49 (2004)

33. Kemper, P.R., Dupuis, N.F., Bowers, M.T.: A new, higher resolution, ion mobility mass spectrometer. Int. J. Mass Spectrom. 287, 46-57 (2009)

34. Srebalus, C.A., Li, J., Marshall, W.S., Clemmer, D.E.: Gas phase peparations of electrosprayed peptide libraries. Anal. Chem. 71, 39183927 (1999)

35. Kurulugama, R., Nachtigall, F.M., Lee, S., Valentine, S.J., Clemmer, D. E.: Overtone mobility spectrometry. Part1. Experimental observations. J. Am. Soc. Mass Spectrom. 20(5), 729-737 (2009)

36. Valentine, S.J., Stokes, S.T., Kurulugama, R., Nachtigall, F.M., Clemmer, D.E.: Overtone mobility spectrometry. Part 2. Theoretical considerations of resolving power. J. Am. Soc. Mass Spectrom. 20(5), 738-750 (2009)

37. Lee, S., Ewing, M., Nachtigall, F., Kurulagama, R., Valentine, S., Clemmer, D.E.: Determination of cross sections by overtone mobility spectrometry: evidence for loss of unstable structures at higher overtones. J. Phys. Chem. B 114(38), 12406-12415 (2010)

38. Glaskin, R., Valentine, S.J., Clemmer, D.E.: A scanning frequency mode for ion cyclotron mobility spectrometry. Anal. Chem. 82(19), 8266-8271 (2010)

39. Mason, E.A., McDaniel, E.W.: Transport properties of ions in gases. Wiley, New York (1988)

40. Shvartsburg, A.A., Jarrold, M.F.: An exact hard-spheres scattering model for the mobilities of polyatomic ions. Chem. Phys. Lett. 261, 8691 (1996)

41. Mesleh, M.F., Hunter, J.M., Shvartsburg, A.A., Schatz, G.C., Jarrold, M.F.: Structural information from ion mobility measurements: effects of the long-range potential. J. Phys. Chem. 100, 16082-16086 (1996)

42. Wyttenbach, T., von Helden, G., Batka, J.J., Carlat, D., Bowers, M.T.: Effect of the long-range potential on ion mobility measurements. J. Am. Chem. Soc. 8, 275-282 (1997)

43. Wittmer, D., Luckenbill, B.K., Hill, H.H., Chen, Y.H.: Electrospray ionization ion mobility spectrometry. Anal. Chem. 66, 2348-2355 (1994)

44. Hoaglund, C.S., Valentine, S.J., Sporleder, C.R., Reilly, J.P., Clemmer, D.E.: Three-dimensional ion mobility/TOFMS analysis of electrosprayed biomolecules. Anal. Chem. 70, 2236-2242 (1998)

45. St Louis, R.H., Hill, H.H.: For a review of IMS techniques see (and references therein): ion mobility spectrometry in analytical chemistry. Crit. Rev. Anal. Chem. 21, 321-355 (1990)

46. Clemmer, D.E., Jarrold, M.F.: For a review of IMS techniques see (and references therein): ion mobility measurements and their applications to clusters and biomolecules. J. Mass Spectrom. 32, 577-592 (1997)

47. Bohrer, B.C., Merenbloom, S.I., Koeniger, S.L., Hilderbrand, A.E., Clemmer, D.E.: For a review of IMS techniques see (and references therein): biomolecule analysis by ion mobility spectrometry. Annu. Rev. Anal. Chem. 1(10), 1-10 (2008)

48. Shaffer, S.A., Prior, D.C., Anderson, G.A., Udseth, H.R., Smith, R.D.: An ion funnel interface for improved ion focusing and sensitivity using electrospray ionization mass spectrometry. Anal. Chem. 70, 4111 (1998)

49. Him, T., Tolmachev, A.V., Harkewicz, R., Prior, D.C., Anderson, G., Udseth, H.R., Smith, R.D., Bailey, T.H., Rakov, S., Futrell, J.H.: Design and implementation of a new electrodynamic ion funnel. Anal. Chem. 72, 2247 (2000)

50. Julian, R.R., Mabbett, S.R., Jarrold, M.F.: Ion funnels for the masses: experiments and simulations with a simplified ion funnel. J. Am. Soc. Mass Spectrom. 16(10), 1708-1712 (2005)

51. Dahl, D. A. SIMION (Version 7.0); Idaho National Engineering Laboratory: Idaho Falls, ID 